\title{
Extending Learning Beyond the Planetarium with the Dome+ Model
}

\author{
Jesica L. Trucks \\ Abrams Planetarium/Department of Physics and Astronomy, \\ Michigan State University, East Lansing, Michigan, 48824 \\ Kathleen A. Hinko \\ Department of Physics and Astronomy and Lyman Briggs College, \\ Michigan State University, East Lansing, Michigan, 48824 \\ Shannon Schmoll \\ Abrams Planetarium, Michigan State University, East Lansing, Michigan, 48824
}

Planetarium visits limit the amount of time and agency people have to explore content relevant to their interest and identities as compared to other informal learning environments. We developed the Dome+ model to guide extending engagement beyond planetariums based on the design principles of identity, interest, and choice. We do this through web resources, social media, and hands-on activities centered on a planetarium show. We present research into the development and implementation of the Dome+ model as it applies to the Big Astronomy Project. This is a multi-institutional effort to share the stories of the people and places that make astronomy happen. The work presented focuses on the implementation of the model during production of the planetarium show. We interviewed team members key in the production of the show and find themes that differ based on project role. We also discuss practical challenges in implementing the Dome+ model. 


\section{MOTIVATION}

Informal astronomy education is primarily conducted at planetariums, which are dedicated physical immersive theaters [1]. Planetarium shows, especially those offered for free or low cost for planetariums, reach a broad audience and support educating the public. For instance, the very low cost show Back to the Moon For Good reached 287 planetariums in the United States and 900 globally, with an estimated global viewership of more than 8 million people [2].

Experiences in the planetarium are limited by the short amount of time people are in a planetarium and the lack of choice and control audiences have as compared to other informal learning environments (ILEs) [3]. These limitations are problematic because people need multiple exposures to concepts over extended periods of time to effectively learn new concepts $[4,5]$. Since multiple visits are not always possible for visitors in ILEs, it is important to create a means of supporting learning beyond their physical footprint and create other opportunities for exposure. Research in astronomy education that has looked at learning "beyond the dome" has almost always looked at how planetarium content can integrate into formal curriculum (such as K-12 or college) [3, 68]. Web and mobile-based means of extending learning have been studied within the context of museums [9, 10] and are predicated on the key characteristic of choice and control in one's learning, allowing visitors to personalize their experience online based on their existing interests. There is little to no research that supports extended engagement in the context of planetariums with a general audience.

In this paper, we present a new model for planetarium education that seeks to expand the impact of planetarium shows by integrating additional activities and resources that build upon show content - we call this the Dome+ model. We discuss theories of learning that inform the model and the design principles that define it. We then describe the set of resources that we have developed that codify the design principles into activities that connect to planetarium shows. Specifically, we describe the development of our first implementation of the Dome+ model in the "Big Astronomy" project, which focuses on careers at major observatories in Chile.

In order to document putting this model into practice, we have collected interview data with different members of the Big Astronomy team to understand how they have built resources using the Dome+ model design principles. This paper focuses on the creation of the planetarium show itself within this model and team members who worked on this part of the project. We have analyzed these interviews using a grounded theory approach and present initial findings on the key themes and challenges in putting this new model into practice.

\section{THEORIES OF INFORMAL LEARNING}

The National Research Council report on informal STEM learning [11] identifies two goals that differentiate informal from formal learning - 1) building interest and 2) developing scientific identity. Interest is key in ILEs because of the time limitations visitors have. It is important to engage people enough that their interest is piqued and they wish to learn more later. For people to pursue interests in STEM, they must also see themselves as someone who can participate. This identification is important for people to pursue hobbies, take an informed position on public policy, and employ scientific thinking in everyday life. If students identify with doing science, they are more likely to remain motivated through difficult tasks [12]. Building STEM identity and representation is not enough. People have different identities that they are building and tapping into [13]. People also identify with their culture, their language, other interests, and so on [14]. So it becomes important to support science learning in a multitude of different ways to mesh with the many ways that people see themselves and allow fluidity among these different identities.

Because people have different interests and identities, they learn differently. Identities can inform interests and vice versa. ILEs support identity development by giving people agency over their own learning, often more so than formal environments[15]. However, planetariums offer far less choice for an individual than museums or other ILEs. To experience agency in their learning, a visitor must actively choose to learn more beyond the dome.

\section{THE DOME+ MODEL}

Our goal with the Dome+ model is to extend engagement beyond the dome through design around the critical constructs of agency, identity, and interest. In Dome+, these constructs become principles for the design of resources which support learning goals. Learning goals are the educational outcomes related to specific show content.

In the model, we keep the planetarium show as the central resource but look to create opportunities for engagement once people leave the dome through related resources. We propose three additional categories of resources: web portal, strong social media presence (particularly through live events), and hands-on activities. We postulate these resources could provide people choices in how to engage that fit their lives, interests, and identities the best and make it easier for them to choose to learn more. Figure 1 shows how both the Dome+ model design principles and the content-specific learning goals inform the suite of concrete resources that support the show.

The Dome+ model is intended to support agency by allowing multiple avenues to engage both online and offline and in different locations. Implementation of the model should support interest by allowing people to follow different pathways through the resources based on what interests them the most within the additional content areas and types of content. 


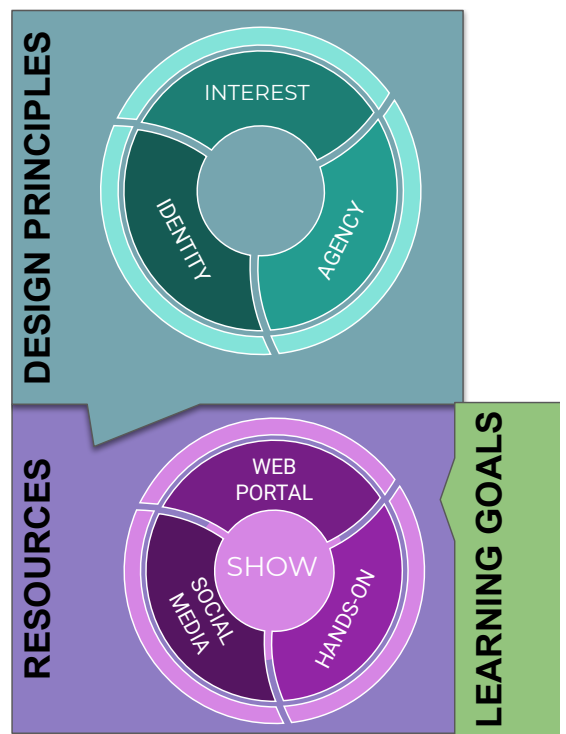

FIG. 1. The Dome+ Model

\section{A. Using the Dome+ Model with Big Astronomy}

The first implementation of the Dome+ model is centered on the bilingual planetarium show Big Astronomy: People, Places, and Discovery about large-scale astronomy research in Chile. The Big Astronomy Project seeks to support identity by featuring people from various backgrounds performing diverse jobs. One objective is to show people who work in observatories as whole people who integrate multiple identities with their STEM identities.Here we describe our specific learning goals and the resources developed using the design principles.

Audiences will learn about: 1) Importance of teamwork, which refers to the diverse skills and collaborations required to make scientific discoveries happen; 2) Chile's climate and geography make it an ideal location for observational astronomy; 3) Astronomy is a multimessenger endeavor that uses telescopes that observe at different wavelengths of light to provide complementary views of astronomical phenomena; 4) Modern astronomy has an active connection to the past through indigenous knowledge.

The central resource in the Dome+ model for engaging audiences around content is the planetarium show. The planetarium show was developed over the course of a year. Multiple trips to Chile were taken by a production crew to obtain footage of the outside and inside of key observatories. Interviews were done with observatory staff and community leaders where the observatories are located. Editing took place over the period of several months. The final planetarium show takes viewers around Chile to three major observatories: Cerro Tololo Inter-American Observatory, the Gemini South 8.1-m telescope, and last to the ALMA radio observatory. At each location two to three observatory staff are featured as they talk about their job. Careers highlighted include electronics engineer, telescope operator, telescope mover operator, and a leader from a local indigenous community.

The planetarium show leads to the web portal, which will include additional resources, additional interviews with observatory staff, video content, archived live events, an educator guide, and links hands on activity guides. There will be 52 live social media events spread over a two year period that will feature observatory staff, recent discoveries, live tours of the facilities, and demos of hands-on activities. The kits will include six activities for people to learn more about the content and people featured in the planetarium show. A majority of these kits will also be reproducible at home at little cost. One hundred activity kits will be distributed to educators in astronomy clubs, museums, and planetariums.

Each resource is designed to support the others. Visitors to the show will receive a postcard that features an activity related to the show and a link to the web portal and social media accounts. The show has a call to action in the credits to encourage people to visit the web portal. All show presenters are also encouraged to do a live call to action. The web portal has links to all social media accounts and a map of planetariums showing the show and which venues and groups have access to a hands on kit. The kits will feature giveaways and links on several items back to the web portal.

\section{METHODS}

In this paper, we have thus far presented a new theoretical model for informal planetarium education by extending engagement beyond a show. We have also described the development of the Big Astronomy Project from the Dome+ model, which is informed by learning goals around astronomy in Chile. The Big Astronomy project is nearing the end of the development phase and is ready to move into the dissemination phase for the planetarium show, where it will be shown in planetariums around the US, Chile, and globally. The planetarium show is the first resource to be completed, as was necessary due to time constraints from the production team.

At this point, we find it useful to ask the research questions: How have different Big Astronomy team members executed the Dome+ model within their role on the project? What are different team members' vision/goals for the project? By asking these questions, we are looking to understand how the Dome+ model influenced the decisions of the practitioners in the project. Future work will include looking at the implementation of the suite of resources and audience impact; however, at this stage we focus our research on the development process. In this way, we can learn lessons for groups who seek to implement the Dome+ model with other types of scientific content.

The Big Astronomy project team consists of over 20 people across 7 institutions, and is divided into subgroups focused on the planetarium show, social media/live events, hands-on activities, the web portal, and research/evaluation. 
The planetarium show is currently completed which allows us to investigate the research questions with respect to this particular resource. We interviewed four people from the project team, the producer (who performed the interviews with observatory employees featured in the planetarium show), a member of the show production team (who edited the show and created animated sequences), the head of research (who collaborated on the Dome+ model), and the principal investigator of the project (who coordinates between all groups on the project). We used a semi-structured interview protocol for these interviews, asking team members about their understanding of the Dome+ model, their process in creating the resources, how the Dome+ model factored into their process and decisions, and what they would change if they employed the Dome+ model on a future project. The interviews ranged from approximately 15 to 30 minutes in length. As a note, the Head of Research was also involved with the creation of the interview protocols.

We utilized a grounded theory approach wherein the theory is generated from the researcher's observations and interviews [16]. We listened to each interview multiple times, writing down time stamps and notes of observations about what they said and how that related to the model, and how the model was implemented by each team member. These notes revealed themes from which codes were generated and discussed within the research team, shown in Table I. Each interview was coded by at least two team members. After coding we compared codes and insights between members of the research team. Our final codes counts which were in $100 \%$ agreement amongst the research team are shown in Table II. The final code counts are separated by team member interviews.

The emergent codes fall into three main categories - codes about the Dome+ model, codes about the design principles of interest and identity, and codes about challenges in the project. Descriptions and examples of these codes from the data are shown in Table I.

\section{ANALYSIS}

The analysis of the interviews revealed a divergence in themes based on the team member's role in the project. Identity and interest were prevalent in the interviews with the production staff members whose work was focused solely on the planetarium show. Interviews with the Principle Investigator (P.I.) and Head of Research focused more on the larger unified ideas of the model.

The interviews with the P.I. and Head of Research spoke in a comprehensive manner about the Dome+ model which can be shown by the code counts in Table II. The P.I. stated that within the Dome+ model "what we are working to develop is a process and tools that can be replicated in the future that ultimately lead to getting people engaged initially at some level[...] ]and creating the structure that facilitates additional and future engagement." The Head of Research stated that within the Dome+ model "whatever we are designing we are addressing [the design principles] in some way." She talked about the resources as needing to support interest, support a STEM identity and show through role models that a STEM identity can be integrated with multiple identities, and that people are given a choice in how they engage their interest and how they express their identity.

Different themes were the focus in the interviews with show production members. The show production team members mostly spoke around identity and interest (see Table II). The producer of the show who interviewed all of the "stars" of the planetarium show spoke about her process in deciding who she chose for interviews and wanting diversity in their identities to create role models for visitors. She stated that the main goal was "inspiring people into STEM jobs that might not think that there would be room for them." The show production member was working with the show director and producer to create the story. He was focused on finding the story for the show and said that one instance in particular came about when he "was able to meet with a couple of fellas who worked in the electronics lab and they showed me a bunch of their stuff and it was just really interesting and so we ended up working that into the show." He was also focused more on the video production end of the show and talked about the importance of finding "beautiful" shots.

A consistent theme across nearly all of the interviews were around challenges in producing the show. All but the producer noted the logistical challenges of producing the show and working in a very large team across different states. Also, the production team for the show had a very tight schedule in which to film the show at the project locations that caused challenges. The specific nature of the logistical challenges from the editor were around working on a show in a different country and in a different language, that made getting the work done in a timely manner more challenging. The PI and Research Lead expanded upon the limited time the production team had to finish the show and how that affected the implementation of the model.

Both the PI and the research lead noted that time limitations surrounding the show production meant that there was not enough time to fully develop the model and implement it before the show production started. Specifically, the research lead noted "I think we could have had that time to more thoughtfully plan out those connections from the beginning". This resulted in the show not benefiting from the model as effectively.

\section{DISCUSSION AND FUTURE WORK}

Planetariums do not offer choice in the same way as most informal learning environments. So we need to provide choice through other additional resources for extended engagement. Here we have presented the Dome+ model, which designs for agency, identity, and interest in the context of learning goals through a suite of additional resources. 
TABLE I. Code Descriptions and Examples

\begin{tabular}{|c|c|c|}
\hline Code & Description & Example Quote \\
\hline dome+DP & $\begin{array}{l}\text { design principles as a whole: } \\
\text { agency, identity, and interest }\end{array}$ & $\begin{array}{l}\text { "informal learning creates interest for people, it generates interest for learning } \\
\text { more, }[\ldots] \text {, one of the strengths of informal learning is they can choose to learn } \\
\text { more, }[\ldots] \text {, we want to support STEM identity through what we are doing." }\end{array}$ \\
\hline dome $+\mathrm{R}$ & $\begin{array}{l}\text { resources that support design } \\
\text { principles and learning goals }\end{array}$ & $\begin{array}{l}\text { "The planetarium show was meant to sort of be the kickoff for the web portal [...] } \\
\text { where we have all sort of supplemental educational materials." }\end{array}$ \\
\hline dome+Unity & $\begin{array}{l}\text { how resources and design } \\
\text { principles support one another }\end{array}$ & $\begin{array}{l}\text { "how is this network [of resources] facilitated and how we are putting in the roads } \\
\text { that connect all of the different pieces" }\end{array}$ \\
\hline interest & generating interest in visitors & $\begin{array}{l}\text { "in a lot of these [shooting locations] we were going for the most beautiful shots } \\
\text { we could get" }\end{array}$ \\
\hline identityDiv & $\begin{array}{l}\text { diversity in identities that are shown } \\
\text { to visitors }\end{array}$ & $\begin{array}{l}\text { "for me it was a lot about diversity, not just diversity of people but also the jobs } \\
\text { they did [...] all sorts of different people with all sorts of different backgrounds" }\end{array}$ \\
\hline identityEvo & $\begin{array}{l}\text { evolution or development of } \\
\text { identities shown to visitors }\end{array}$ & $\begin{array}{l}\text { "really gave an insight into her personality and I think would help others [...] } \\
\text { [show] how they would get beyond that so that their dreams are not shut down } \\
\text { because they are struggling" }\end{array}$ \\
\hline challengeLog & logistical challenges in the project & $\begin{array}{l}\text { "because we were there for production a short window of time }[\ldots] \text { opportunities } \\
\text { [that] were available [drove] what we were shooting" }\end{array}$ \\
\hline challengeTeam & $\begin{array}{l}\text { challenges when working with a } \\
\text { large team in diverse locations }\end{array}$ & "would have been nicer would be to have spent more time with the whole team" \\
\hline
\end{tabular}

TABLE II. Code Counts

\begin{tabular}{ccccc}
\hline \hline Code & P.I. & $\begin{array}{c}\text { Research } \\
\text { Lead }\end{array}$ & Producer & Editor \\
\hline dome+DP & 2 & 7 & 0 & 0 \\
dome+R & 1 & 5 & 1 & 2 \\
dome+Unity & 5 & 4 & 0 & 0 \\
interest & 1 & 1 & 0 & 4 \\
identityDiv & 0 & 1 & 3 & 1 \\
identityEvo & 1 & 5 & 4 & 2 \\
challengeLog & 4 & 4 & 0 & 3 \\
challengeTeam & 4 & 3 & 0 & 2 \\
\hline \hline
\end{tabular}

Putting a model into practice though, is constrained by the humans who are doing that work and logistics surrounding development. We document how the team members of the Big Astronomy project took these key design principles up in different ways with respect to the planetarium show resource. The P.I. and Head of Research focused more on the Dome+ model and its implementation as a whole, while show production members were focused on identity and interest. From this finding, we see that the design principles of identity and interest are valued and promoted within the show. It is not surprising that agency was not discussed by the production team members, as they are limited by the format of the planetarium show. There were also challenges around the production team needing to immediately begin work, not allowing the team to appropriately influence the show production through the model. From these results, we suggest it might be useful for teams to be organized so all team members are well versed in the Dome+ model, in order to help the show connect strongly to all the design principles and also to the other resources in the model in future implementations of Dome+.

This work here is just the beginning of a larger research project. Future work will look at the implementation of more resources of the project. This work focused mostly on the planetarium show as it is complete and has begun distribution. The research of the full implementation will include interviews with visitors to planetariums, and planetarium directors and personnel, field notes from planetariums site visits, surveys, data analytics from the web portal and social media accounts, and public social media data related to the live events. The launch of the full implementation of the project has been delayed by the current pandemic and therefore future work may include adjusting the project to understand the virtual components in the era of COVID-19. Longer term goals include developing this model more robustly, making it adaptable to content and learning goals, and applying it to future planetarium show productions.

The Big Astronomy Project is funded by NSF award \#1811436. The Big Astronomy Project Team is composed of members from Associated Universities, Inc., Association of Universities for Research in Astronomy, Michigan State University, California Academy of Sciences, Astronomical Society of the Pacific, Peoria Riverfront Museum, and Ward Beecher Planetarium. 
[1] J. A. Percy, ed., The Role of Planetariums in Astronomy Education (Astronomical Society of the Pacific (ASP), 1996).

[2] Back To The Moon For Good. Personal Communication with Project's Leadership. https://www.fddb.org/fulldomeshows/back-to-the-moon-for-good/.

[3] S. Schmoll, Toward a framework for integrating planetarium and classroom learning., Unpublished Dissertation. University of Michigan (2013).

[4] J. Minstreall, Teaching (Association for Supervision and Curriculum Development, Alexandria, VA, 1989) Chap. Teaching science for undestanding, pp. 129-149.

[5] G. Posner, K. Strike, P. Hewson, and W. Getzog, Accomodation of a scientific conception: toward a theory of conceptual change, Science Education 66 (1982).

[6] J. D. Plummer and K. J. Small, Integrating Planetarium and Classroom Instruction to Engage Children in the Practices of Science, in Ensuring Stem Literacy: A National Conference on STEM Education and Public Outreach, edited by Astronomical Society of the Pacific Conference Series, Vol. 483J. G. Manning, M. K. Hemenway, J. B. Jensen, and M. G. Gibbs (2014) p. 407.

[7] L. Lelliot, Learning about astronomy: a case study exploring how grade 7 and 8 students experience sites of informal learning in South Africa, Ph.D. thesis, University of Wiwatersrand, Johannesburg, South Africa (2007).

[8] A. R. Sarrazine, Addressing Astronomy Misconceptions and Achieving National Science Standards Utilizing Aspects of Multiple Intelligences Theory in the Classroom and the Planetarium, $\mathrm{Ph} . \mathrm{D}$. thesis, Indiana University, Bloomington, Indiana (2005).
[9] C. Cahill, S. Schmoll, I. Delen, W.-T. Lo, A. Kuhn, B. McNally, and J. ... Krajcik, Bridging inquiry across settings using mobile and curricular supports.presented at the annual international conference of the national association for research in science teaching (2012).

[10] A. L. approach to evaluating MyArtSpace, Giasemi vavoula and julia meek and mike sharples and peter lonsdale and paul rudman, (2006).

[11] NRC, edited by Learning Science in Informal Environments: People, Places, and PursuitsP. Bell, B. Lewenstein, A. W. Shouse, and M. A. Feder (National Academies Press, 2009).

[12] M. R. Lepper and J. Henderlong, Chapter 10 - turning "play" into "work" and "work" into "play": 25 years of research on intrinsic versus extrinsic motivation, in Intrinsic and Extrinsic Motivation, edited by Educational PsychologyC. Sansone and J. M. Harackiewicz (Academic Press, San Diego, 2000) pp. 257 -307 .

[13] Z. Hazari, G. Sonnert, P. M. Sadler, and M.-C. Shanahan, Connecting high school physics experiences, outcome expectations, physics identity, and physics career choice: A gender study, Journal of Research in Science Teaching 47, 978 (2010), https://onlinelibrary.wiley.com/doi/pdf/10.1002/tea.20363.

[14] M. Shanahan, Identity in science learning: exploring the attention given to agency and structure in studies of identity, Studies in Science Education 45, 43 (2009).

[15] J. H. Falk and L. Dierking, Learning from Museum: Visitors experiences and their making of meaning. (Altamira Press, Walnut Creek, CA, 2000).

[16] M. Q. Patton, Qualitative Research and Evaluation Methods, 3rd ed. (Sage, 2002). 